\title{
FREE ELECTRON LASERS WITH ELECTRON STORAGE RINGS
}

\author{
N.A. Vinokurov \\ Institute of Nuclear Physics, 630090 Novosibirsk, USSR
}

Abstract. The basic physical limitations on the parameters of FELs installed at electron storage rings have been described. The prospects of these devices have been discussed, and the results of the recent experiments have been reported.

\section{Introduction}

Free electron lasers are the devices where the energy of a relativistic electron beam converts to coherent electromagnetic radiation when the particles pass through a special magnetic system with an alternating periodic magnetic field (undulator). The appropriate electron beam are produced using accelerators of various types.

In the case of using electron storage rings, the $\mathrm{FEL}$ undulator is positioned in a straight section and one and the same electrons pass periodically through the FEL and interact here with radiation. Such a situation gives rise to two attractive peculiarities. First, this is a high average power (the product of the average current by the energy divided by the electron charge) of the beam, which is reactive for this case. For example, at an energy of $1 \mathrm{GeV}$ and a current of $0.1 \mathrm{~A}$ we obtain the power equal to $100 \mathrm{MW}$. The production of beam of such power on the other types of accelerators is very complicated and rather expensive. Second, owing to radiation damping in electron storage rings, beam emittances transverse and longitudinal) can be fairly small, thereby allowing the creation of short-wavelength FELs (the ultraviolet range inclusive). On the other hand, these advantages are likely to be illusory. Multiple interactions of one and the same electrons with radiation lead to sheating» the electron beam and to a specific restriction of radiation power, and, as a consequence, the first advantage is brought down to a minimum. Progress in the development of electron guns (in particular, guns with photocathodes) has provided the production of beams with a brightness considerably higher than that of the beams generated by storage rings. On the other liand, the FEL on an electron storage ring is the shortest wavelength FEL and a single tunable $\mathrm{CW}$ laser in the ultraviolet. This allows us to hope that the building up of dedicated (optimized for this problem) storage rings will offer the possibility to design FELs of this type, which are applicable to science, technique, medicine, and other field of activity.

In the present paper, we describe the basic physical restrictions on the parameters of FELs on electron storage rings, the possible consequences of these restrictions, as well as the current status of FEL works.

\section{Restrictions in Power}

We will consider a free electron laser installed at the straight section of an electron storage ring. Assuming the main iniormation known from FEL and cyclic accelerator physics, we will confine ourselves to some specific effects occurring in such devices. This situation is characterized by multiple passage of one and the same electrons through a FEL and a high duty factor $(100-1000)$ of the electron current.

Due to the interaction of electrons with the radiation field, in the optical cavity, the energy of outcoming electrons proves to be modulated with the frequency of the optical radiation. For a «weak field in the resonator (this is the case realized in storage rings FELs), an appropriate addition to the electron energy is insignificant as compared with the energy spread $\sigma$ in the electron beam. Because of the smallness of the radiation wavelength $\lambda \ll \alpha \Pi \frac{\sigma}{E}$ ( $\Pi$ is the circumference of a storage ring, $E$ is the mean electron energy, and $\alpha$ is the momentum compaction factor), the energy and the longitudinal coordinate of the beam particles stop to correlate nearly completely by the moment of the next beam-radiation interaction in the FEL. In this case, the energy spread grows diffusively. Radiation damping of synchrotron oscillations gives rise to the equilibrium state. For the optimized parameters of the device, a simple estimation of the maximum average power can be made $[1-4]$ :

$$
P \approx P_{s k} \frac{\sigma_{\max }}{E}
$$

where $P_{\mathrm{SR}}$ is SR power and $\sigma_{\max }$ is the maximum admissible energy spread.

\section{Prospects}

Now we will discuss the prospects of FELs at storage rings (see also [5]). The most important problem is the limitation on average power (1). It is possible to make an assumption that with the magnetic system of the storage ring properly designed, $\sigma_{\max } / E$ can reach $1 \%$. For increasing the SR power, it is desirable to rise the electron energy $E$, because the losses in electron energy per turn, $W$ are srongly dependent on $E$ :

$$
W=\frac{4 \pi}{3}\left(\frac{E}{m c^{2}}\right)^{3} \text { ere } H \text {. }
$$

where $r_{p}$ is the classical electron radius, and $H$ is the field in the storage ring magnets (for simplicity, the field is assumed to be equal in all magnets). Three more arguments speak for the increase in energy. First, as the energy grows, the current thresholds of the instabilities of the electron beam also grows strongly, thus allowing an increase of the electron current $l$. Second, the probability of intrabeam electron scattering (Toushek effect) decreases and, hence, the contribution of this process to the inverse lifetime of electrons becomes less. Third, the scattering of electrons by the atoms of the residual gas also contributes to the inverse lifetime to a less extent, and this makes it possible to decrease the aperture of the vacuum chamber. The small aperture of the vacuum chamber at the location of the FEL permits one to improve the parameters of the magnetic and electrodinamical systems of free electron lasers.

A conventional storage ring usually consumes more than $1 \mathrm{MW}$ (from the mains). Modern r.f. systems are 
able to transfer $P_{\mathrm{SR}}=1 \mathrm{MW}$ to the circulating electron beam. In this case, the radiation power of a FEL will be about $10 \mathrm{~kW}$ and, thus an efficiency from the mains is about $0.1 \%$. Assuming the average current in the storage ring equal to $1 \mathrm{~A}$, we obtain $W=1 \mathrm{MeV}$. Using eq. (2) at $H=18 \mathrm{kG}$ (nonsuperconducting magnets), we have $E=2.8 \mathrm{GeV}$, while at $H=60 \mathrm{kG}$ (superconducting magnets) $E=1.8 \mathrm{GeV}$. The magnetic system of the storage ring VEPP-3, which was built at the INP 20 years ago is an illustration of the first-type magnetic system [6]. The magnetic system of the second type may be illustrated by that which is developed for the SR source indended for X-ray angiography [7]. Both machines are rather compact: the overall dimensions of VEPP-3 are $18 \times 30 \mathrm{~m}$, and those of the superconducting storage ring are a little less.

There are, however, two circumstances not allowing further increase of the electron energy. The first is the increase in the horizontal transverse emittance of the electron beam $\varepsilon_{x}$. This sets a lower bound on the range of FEL wavelengths: $\lambda>2 \pi \varepsilon_{x}$. For VEPP-3, $\varepsilon_{x}=$ $=0.7 \cdot 10^{-1} \mathrm{~cm} \lambda>4 \mu \mathrm{m}$ at an energy of $3 \mathrm{GeV}$ corres. ponding to $1 \mathrm{MeV}$ losses per turn. For the superconducting storage ring $\lambda>0.1 \mu \mathrm{m}$. The above magnitudes of the emittances refer to the particular magnetic systems. Optimization of the magnetic systems of the storage rings, which is aimed at minimizing the emittance $[8,9]$, enables (at the expense of a certain complication of the magnetic lattice) the emittance to be reduced by a factor of tens and the limitation on the energy growth in such storage rings to be eliminated.

The second limitation is connected with the fact that the wavelength of a fundamental harmonic of undulator radiation on the electron energy

$$
\lambda=\frac{d}{2 \gamma^{2}}\left(1+K^{2}+\frac{1}{2} \theta^{2} \gamma^{2}\right)
$$

where $\gamma=E / m c^{2}$ is the relalivistic factor, $\theta$ - the angle at which radiation is observed, $d$-the undulator period.

$$
K=\frac{e d}{2 \pi m c^{2}} \sqrt{\left\langle H^{2}\right\rangle}
$$

the deflection parameter, and $\left\langle H^{2}\right\rangle$ - the square of the transverse magnetic field which is averaged along the undulator axis. The axis of the optical resonator in a FEL, the undulator axis and the mean orbit of electrons in the undulator usually coincide. In this case, $\theta=0$, and for the given wavelength $\lambda$ and electron energy $E$ we derive the condition for the parameters of an undulator:

$$
d\left(1+K^{2}\right)=2 \gamma^{2} \lambda
$$

It is clear that at high energies the condition (5) is reasier for short wavelengths $\lambda$ and, hence, we choose $\lambda=0.1 \mu \mathrm{m}$. For shorter wavelengths, the radiation absorbs in a substance considerably more intense, thus making impossible (at least, for the time being) the fabrication of mirrors for the FEL optical resonator with a reflectivity close to unity. At $E=1.8 \mathrm{GeV}$, we obtain $d\left(1+K^{2}\right)=2.5 \mathrm{~m}$. This is easy to be done, for example, at $d=0.1 \mathrm{~m}$ and $\sqrt{\left\langle H^{2}\right\rangle}-5.2 \mathrm{kG}$. So, large $K$ are required for the FEL performance at high energy. As known $[10,11]$, the power of spontaneous emission on the harmonics of the fundamental wavelength $(3)$ is relatively high at large $K$; note that for $K \gg 1$ and large harmonic numbers this radiation converts to a conventional SR. Arriving at the front mirror of the optical resonator, such radiation spoils the mirror (for details see Refs 12 and 13). At high electron energies, high $K$ and electron currents, the situation dramatically worsens. One of the possibility way out is to use helical undulators [14]. The bulk of the radiation power from $\theta$ such undulators is concentrated near $\theta=K / \gamma$. The radiation on the fundamental harmonic is concentrated in the vicinity of $\theta=0$ with the angular spread $\frac{1}{\gamma} \sqrt{\frac{1+K^{2}}{N}}$ ( $N$ is the number of undulator periods). which is considerably less than $K / \gamma$ at $N \gg 1$. Thus, it is possible to provide conditions under which only a «useful portion of radiation arrives at a mirror. So, we see that the conditions (5) can be fulfilled for short waves, but sets an upper bound on the range of wavelengths.

To extend this range, the electron energy can be somewhat reduced compensating the decrease in energy losses per turn. For this purpose, either additional superconducting wigglers could be installed at the equilibrium orbit of the storage ring, or the major magnetic system with an alternating magnetic field could be used. For example, to decrease the storage ring energy down to $1 \mathrm{GeV}$, it suffices either to position a 20-m-long superconducting wiggler with a $60 \mathrm{kG}$ r.m.s. field or a four-fold increase of the number of bending magnets in the superconducting storage ring. For the long-wave radiation to be generated, we may use the $\theta$-dependence of $\lambda$ in (3). For cxample, we insert a wavequide inside the undulator (let the wavequide comprise two conducting planes which are $a$-distant from each other). For the lowest $H$-wave, we have $\theta=\lambda / 2 a$. At $\gamma \rightarrow \infty$, from eq. (3) we obtain $\lambda=4 a^{2} / d$. Another possibility to generate long-wave radiation is to use Cherenkov FEL $[15,16]$ (to be more precise, Cherenkov optical klystron to provide electron bunching at high energy).

\section{Experimental Results}

We will consider briefly the basic results of FEL experiments. In 1983 , the induced radiation was first generated using an optical klystron at the storage ring ACO (Orsay, France) [17]. The average power achieved at this device is $30 \mathrm{~mW}$ (we present the power taken away from the electron beam) and the regime of giant pulses was demonstrated. The coherent radiation was produced in the $0.655-0.463 \mu \mathrm{m}$ range. In this case, the generation bandwidth was about $1 \AA$. In addition, experiments were made on generation of the radiation harmonics of an outer solid laser at the $0.177 \mu \mathrm{m}$ and $0.106 \mu \mathrm{m}$ wavelengths. A detailed description of these experiments may be found in $[18]$.

In 1988, the induced radiation generation was obtained using the bypass of VEPP-3 within the $0.69-0.24 \mu \mathrm{m}$ range. Further shortening of the wavelength is limited only by the absence of the appropriate mirrors. The maximum average power was roughly equal to $5 \mathrm{MW}$. The duration of light pulses (about $0.2 \mathrm{~ns}$ ) was measured with a dissector [19] and obser. vations wcre made concerning the change of the longitudinal electron distribution in the bunch as a result of the interaction between the electrons and the radiation in the optical resonator. Thus, the measured increase in the energy spread up to $\sigma / E=1 \cdot 10^{-3}$ is in agreement 
with the calculated one and with the value of the radiation power. In the regime of giant pulses generation we obtained the maximum mean-in-macropulse power equal to $20 \mathrm{~W}$ for red light $(0.63 \mathrm{um})$ and to $5 \mathrm{~W}$ for the ultraviolet $(0.25 \mathrm{um})$. Taking into consideration that the repetition frequency is $8 \mathrm{MHz}$, i. e. the duty factor is 625 , we obtain the peak powers equal to $12 \mathrm{~kW}$ for red light and to $4 \mathrm{~kW}$ for the ultraviolet. The snatural» bandwidth of radiation was $0.5 \AA$. After a glass plane-parallel plate of $1.2 \mathrm{~mm}$ thick was placed inside the optical resonator, we succeeded in narrowing the band to $0.03 \AA$ (without any substantial reduction in power). The results obtained are well consistent with the calculated. A careful description of the work we have done is presented in $[20-22]$.

In 1989 the optical klystron installed at a new positron storage ring SUPER ACO was put into operation [23]. For the time being, the experiments are being performed at a single wavelength equal to $0.63 \mathrm{um}$. The mean power obtained amounts to $300 \mathrm{~mW}$. The high energy $(600 \mathrm{MeV})$ contributes to an obtaining of this relatively high power.

We would like to mention the trend towards energy increase in storage rings for FELs: the FEL at ACO operated at the energies 150 to $250 \mathrm{MeV}$, at VEPP-3 at $350 \mathrm{MeV}$, at SUPER ACO at $600 \mathrm{MeV}$; In the USA and West Germany, dedicated storage rings for FELs at $1-1.5 \mathrm{GeV}$ energy are being constructed $[24,25]$.

\section{Conclusion}

So, we see that a gap in power between the existing FELs at storage rings and those of the nearest future is four orders of magnitude. This is not so much if account is taken of the fast today's FELs are installed at the storage rings intended for other purposes. The progress in accelerator technique, which is due to the creation of SR sources of «wide use gives the hope that the cust of FELs under discussion will be quite reasonable. The most probable spectral range for such FELs is the visible and ultraviolet ranges of 'wavelengths.

\section{References}

[1] N.A. Vinokurov and A.N. Skrinsky. On Ultimate Power of the Optical Klystron Installed at the Electron Storage Ring.- Preprint INP 77-67, Novosibirsk, 1977.

[2] N.A. Vinokurov and A.N. Skrinsky. On Ultimate Power of the Optical Klystron. Proc. of VI National Meeting on Charged Particle Accelerators, 1980, v.2, p.233.

[3] A. Renieri. «Storage Ring Operation of the Free Electron Laser the Amplifiers, Nunvo Cimento, 1979, v.B53, 160
(4) G. Dattoli. A. Renieri. \& Storage Ring Operation of the Free Electron Laser: the Oscillator», Nuovo Cimento, 1980, v.B59, p. 1

[5] D.A.G. Deacon. «Storage Ring Driven Free Electron Lasers», SPIE, 1987, v.738, p.38.

[6] G.L Budker, I.Ya Protopopov and A.N. Skrinsky. Device with Colliding Electron-Positron Beams at $3.5 \mathrm{GeV}$ (VEPP-3). Proc. of VII Intern. Conf. on Charged Particle Accelerators at High Energies, Yerevan, 1970, y.2, p.37.

[7] V.V. Anashin et al. *Compact Storage Rings Siberia-AS and Siberia-SM Synchrotron Radiation Sources for Lithography». Rev. Sci. Instr., 1989, N 7, v.60, p.1767.

[8] H. Wiedemann. *Storage Ring Design Optimisation for FEL Operation». Proc. of the Intern. Free Electron Laser Conference, Bendor, France, Journ. de Physiqe, 1982, v.44-Cl, p.201.

(9) V.N. Korchuganov et al. EOptimization of Parameters of a Dedicated Synchrotron Radiation Source for Technologys. Nucl. Instr. and Meth., 1983, v.208, p.11.

[10] G.N. Kulipanov and A.N. Skrinsky. Utilization of Synchrotron Radiation; Status and Prospects, Usp. Fiz. Nauk, 1977. v. 122, p. 369 .

[11] I.M. Ternov, V.V. Mikhailin and V.P. Khalimov. Synchrotton Radiation and Applications, Moscow, 1980

[12] P. Elleaume et al. *Diagnostic Techniques and VUV Induced Degradation of the Mirrors Used in the Orsay Storage Ring Free Electron Lasers, Nucl. Instr. and Method, 1985, v.A237, p. 263 .

[13] M. Ambrosio et al. aDielectric Mirrors Damage Due to the Radiation of a High K Undulator*, Nucl. Instr. and Method 1986, v.A250, p. 289 .

[14] D.F. Alferov, Yu.A. Bashmakov and E.G. Bessonow. Undulator Radiation. Trudy FIAN, Moscow, Nauka, 1975, v.80 p. 100

[15] I.E. Walsh, I.B. Murphy. Tunable Cherenkov Lasers», IEEE J. of Quantum Electronics, Aug. 1982, p.1259.

[16] N.F. Kovalyov et al. O-Type Devices Based on Cherenkov and Transient Radiations of Relativistic Electrons. In: Relativistic R.F. Electronics», IPF, Gorky, 1979, p.76.

[17] M. Biltardon et al. *First Operation of a Storage Ring Free Flectron Laser . Phys. Rev. Lett., 1983, v.51, p.1652

[18] M. Billardon. Free Electron Laser Experiment at Orsay: a Review, IEEE J. of Quantum Electronics, 1985, v.QE-21, p.805

[19] E.I. Zinin. «Stroboscopic Method of Electrooptical Picosecond-Resolution Chronography and its Application in Synchrotron Radiation Experiments», Nuclear Instr. and Method, 1983, v.208, p.439.

[20] V.N. Litvinenko. Optical Klystron on the VEPP-3 Bypass-Generation in the Visible Spectral Range and in the Uitraviolet. Thesis, Novosibirsk. INP, 1989.

[21] N.A. Vinokurov et al. Kasing in Visible and Ultraviolet Regions in an Optical k'lystron Installed at the VEPP-3 Slorage Rings, Rev. of Sci. Instr., 1989, N 7, v.60, p.1435.

[22] I.B. Drobyazko et al. cLasing in Visible and Ultraviolet Regions in an Optical Klystron Installed at the VEPP-3 Storage Ring», Nucl. Instr. and Method, 1989, v.A282, p.424.

[23] $R$. Prazers et al. \&Optical Klystron Experiments at Orsay on Super-Aco and Prospects in the VUV», Rev, of Sci. Instr., 1989, N 7, v. 60 , p. 1429 .

[24] J.E. La Sala, D.A.G. Deacon, I.M.J. Madey. *Performance of an XUV FEL Oscillator on the Stanford Storage Ring* Nucl. Instr. and Method, 1986, v.A250, p.262.

[25] K. Wille. Nucl. Instr and Method, 1988, v.A272, p.59 